\title{
Phase competition induced nonlinear elastoresistance effect in thin films of $\mathrm{Pr}_{0.7} \mathrm{Sr}_{0.3} \mathrm{MnO}_{3}$
}

\author{
J. F. Wang and J. Gao ${ }^{\text {a) }}$ \\ Department of Physics, The University of Hong Kong, Pokfulam Road, Hong Kong
}

(Received 15 December 2011; accepted 8 March 2012; published online 26 March 2012)

\begin{abstract}
Thin films of $\mathrm{Pr}_{0.7} \mathrm{Sr}_{0.3} \mathrm{MnO}_{3}$ grown on $0.7 \mathrm{PbMg}_{1 / 3} \mathrm{Nb}_{2 / 3} \mathrm{O}_{3}-0.3 \mathrm{PbTiO}_{3}$ substrates were reversibly strained via the converse piezoelectric effect. The transport properties of $\operatorname{Pr}_{0.7} \mathrm{Sr}_{0.3} \mathrm{MnO}_{3}$ were effectively modulated by the electric fields across the substrate. The roles of strain were explored by measuring resistance as a function of electric field and temperature. For all samples, deviations from a linear resistance-voltage relation were found. Two quantities, area difference and standard deviation, were used to characterize the nonlinearity. Both the nonlinearity and magnitude of resistance modulation peak at temperatures close to those of metal-insulator transition, indicating that the competing phases significantly changes the behaviors of strain responses. (C) 2012 American Institute of Physics. [http://dx.doi.org/10.1063/1.3697687]
\end{abstract}

Perovskite-type manganites have stimulated much attention for decades due to their correlated physics and remarkable properties. ${ }^{1-3}$ These materials are well known for the unexpected sensitivity of resistivity to magnetic fields, namely "colossal magnetoresistance" effect. ${ }^{4,5}$ They are also susceptible to hydrostatic pressure and strain, which can influence both the double exchange interaction and JahnTeller distortion. The effects of strain are usually studied by comparing films with various thicknesses on same substrates or with identical thickness on different substrates. ${ }^{6-8}$ One concern for these two ways is that other factors, such as oxygen nonstoichiometry, ${ }^{9}$ may be considerable. A recently developed route is to reversibly strain the film using piezoelectric substrates. ${ }^{10-20}$ The induced strain usually changes linearly with the voltage across the piezoelectric substrate. ${ }^{21}$ Thus, this method is also suitable to quantitatively determine the impacts of strain. Phase separation is known to be a ubiquitous feature of manganites. ${ }^{1-3}$ To know how phase separation influences the responses to strain is useful for tailoring functional properties. Complex resistance-strain relations were observed in $\left(\mathrm{Pr}_{1-\mathrm{y}} \mathrm{La}_{\mathrm{y}}\right)_{0.7} \mathrm{Ca}_{0.3} \mathrm{MnO}_{3} / \mathrm{Pb}\left(\mathrm{Mg}_{1 / 3} \mathrm{Nb}_{2 / 3}\right)_{0.72}$ $\mathrm{Ti}_{0.28} \mathrm{O}_{3}$. They were interpreted in terms of phase separation. ${ }^{15}$ On contrary, reported resistance-strain relations are simply linear for other manganite films, ${ }^{13,14,16}$ where phase separation should also exist though its scale may be smaller. Hence, more studies are needed to elucidate the real impacts of phase separation on strain response. In this work, we systematically investigate effects of strain in thin films of $\mathrm{Pr}_{0.7} \mathrm{Sr}_{0.3} \mathrm{MnO}_{3}$ (PSMO) using a $0.7 \mathrm{PbMg}_{1 / 3} \mathrm{Nb}_{2 / 3} \mathrm{O}_{3}-0.3 \mathrm{PbTiO}_{3}$ (PMN-PT) substrate. Significant resistance modulation was obtained. Our results indicate that phase competition does not only enhance the sensitivity of resistance to strain but also induce substantial nonlinearity in the relation between resistance and strain.

Thin films of PSMO on (001) oriented $3 \times 5 \times 0.5 \mathrm{~mm}^{3}$ PMN-PT substrates were fabricated using pulsed laser deposition. During deposition, the substrate temperature and oxygen pressure were $680^{\circ} \mathrm{C}$ and $70 \mathrm{~Pa}$, respectively. The film

\footnotetext{
${ }^{\text {a) }}$ Author to whom correspondence should be addressed. Electronic mail: jugao@hku.hk.
}

thickness t, varying from $15 \mathrm{~nm}$ to $100 \mathrm{~nm}$, was controlled by ablation time. Annealing was carried out in situ at deposition temperature in oxygen $\left(10^{5} \mathrm{~Pa}\right)$. Silver $(\mathrm{Ag})$ pads were evaporated onto the surface of PSMO films and the back of PMN-PT substrates as electrodes. The dc voltage across PMN-PT was supplied by a Keithley 6487 voltage source. To limit the possibility of substrate fracture as a result of polarization reversal, most measurements were done in a unipolar manner. The leakage currents in PMN-PT were negligible (less than $10 \mathrm{nA}$ ).

The structural properties of PSMO/PMN-PT were characterized by XRD ( $\theta-2 \theta$ scan). For all samples, no extra peaks other than (001) reflection peaks of PSMO and PMN-PT could be found. The XRD patterns in the vicinity of the PSMO (002) reflection peaks are presented in Fig. 1. As the thickness varies, the peak intensity changes several orders, but the position seems to change very little. The out-of-plane lattice parameters c deduced from these peaks are $\sim 3.830 \AA$, indicating compressive out-of-plane strains $\left[\varepsilon_{\mathrm{zZ}}=\left(\mathrm{c}_{\text {film }}-\mathrm{c}_{\text {bulk }}\right) / \mathrm{c}_{\text {bulk }}\right]$ of $\sim-1 \%$. The in-plane strain $\varepsilon_{\mathrm{xx}}$ can be calculated from $\varepsilon_{\mathrm{xx}}=-\varepsilon_{\mathrm{zz}}(1-\nu) /(2 \nu)$, where $\nu$ is the Poisson ratio. Typical reported $\nu$ for manganites ranges from 0.3 to 0.5 . Thus, the upper limit value of $\varepsilon_{\mathrm{xx}}$ for our PSMO films is $\sim 2.3 \%$ $(\nu=0.3)$. This value is smaller than the lattice mismatch

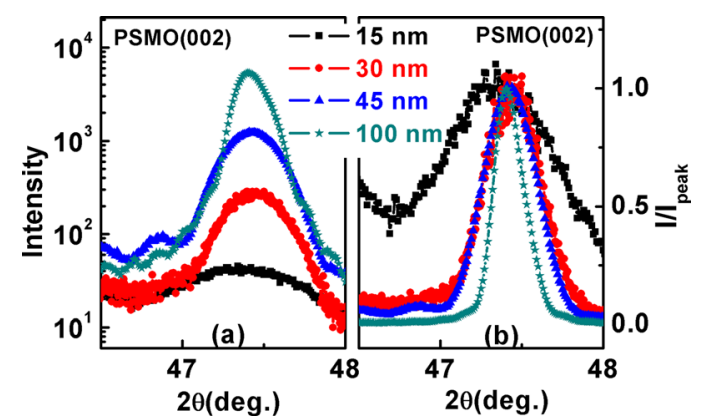

FIG. 1. X-ray diffraction patterns [in the vicinity of (002) reflection peaks of PSMO] for PSMO films with different thicknesses $(t=15,30,45$, and $100 \mathrm{~nm}$ ) on PMN-PT. The curves in (a) are as-measured and in (b) are normalized by the peak intensities. 
between bulk PSMO and PMN-PT $\left[\mathrm{f}_{\text {mis }}=\left(\mathrm{a}_{\mathrm{PMN}-\mathrm{PT}}\right.\right.$ $\left.-\mathrm{a}_{\text {PSMObulk }}\right) / \mathrm{a}_{\text {PMN-PT }} \sim 3.85 \%$ ], signifying that the strain in PSMO is partially relaxed. Although it is not clear how such a relaxation influences the interfacial coupling, efficient modulations have been observed. ${ }^{11-13,15,16}$

The schematic diagram for measurements of transport properties is given in the Fig. 2(a). The polarity shown in the schematic diagram is defined as positive. After PMN-PT had been poled, positive (the same polarity as the poling field) voltages were applied to study the effects of tunable strain. Figures 2(b)-2(d) show the temperature dependences of resistance for PSMO films with different bias voltages on PMN-PT. All PSMO films exhibit metal-to-insulator transition. With the decrease of thickness, the transition temperature $T_{P}$ is suppressed and the resistivity is enhanced. Considering the thickness dependence of XRD pattern, such an evolution of transport properties might be due to the coactions of many factors, for example, strain evolution and finite thickness effect. ${ }^{22}$ For all films, with increasing voltages, resistance was reduced in the whole temperature range and $T_{P}$ moved toward higher temperatures continuously. Under a fixed voltage, the resistance modulation, $|\delta(\mathrm{V})|=|\Delta \mathrm{R}(\mathrm{V})| /$ $\mathrm{R}(0)=|[\mathrm{R}(\mathrm{V})-\mathrm{R}(0)]| / \mathrm{R}(0)$, peaks at a temperature slightly below $\mathrm{T}_{\mathrm{P}}$. Remarkably, for PSMO with a thickness of $15 \mathrm{~nm}$, when the bias voltage reaches $500 \mathrm{~V}$, the maximum $|\delta|$ and the increase of $\mathrm{T}_{\mathrm{P}}$ are as large as $\sim 70 \%$ and $17 \mathrm{~K}$, respectively.

Generally, applying a bias voltage to the poled PMN-PT would simultaneously change the lattice constant in the crystal (via converse piezoelectric effect) and charge density at the surface (via dipole rotation and field effect). Due to the high carrier density and short screening length in doped manganite, the change of charge density usually plays a minor role for manganite/PMN-PT structures. ${ }^{1-14,16,17,20}$ This is verified for our PSMO/PMN-PT by comparing results in positively and negatively poled samples. It is the contraction of in-plane lattice that dominates the effect. As this contraction is transferred to PSMO, it would partially relieve the inplane stretching in PSMO, causing enhanced double exchange interaction and suppressed Jahn-Teller distortion.

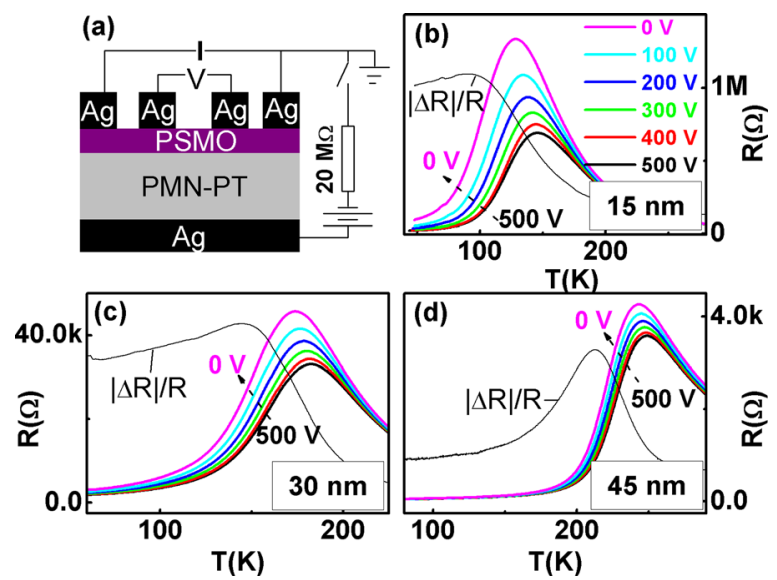

FIG. 2. (a) A schematic diagram of measurement circuit; (b)-(d) R(T) curves for PSMO films with different thicknesses under different bias voltages (from $500 \mathrm{~V}$ to $0 \mathrm{~V}$, the voltage interval is $100 \mathrm{~V}$ ). Temperature dependences of resistance modulation, $|\Delta R| / R=|[R(500 \mathrm{~V})-\mathrm{R}(0)]| / \mathrm{R}(0)$, are also shown.
As a result, $T_{P}$ shifts to higher temperatures and the resistance decreases. It is known that around $\mathrm{T}_{\mathrm{P}}$, there are strong competitions between coexisted multiple phases. ${ }^{1-3}$ The appearance of maximum $|\delta|$ in this region reflects that the tunable strain effectively shifted the balance of competing phases.

We also measured the resistance while cycling voltages at various fixed temperatures (voltage was cycled between $0 \mathrm{~V}$ and $500 \mathrm{~V}$ with a step of $100 \mathrm{~V}$ ). Figures 3(a)-3(c) depict the sequence of resistance as the voltage was cycled for the PSMO film with a thickness of $30 \mathrm{~nm}$ at three selected temperatures. The resistance decreased (increased) as the voltage increased (decreased). As displayed in Fig. 3(d), the dependences of relative resistance change on applied voltages can be deduced from the $\mathrm{R}$-t curves. There is slight hysteresis in the $\Delta \mathrm{R} / \mathrm{R}$ vs $\mathrm{V}$ curves, but the modulation is repeatable. If the resistance changes linearly with applied voltages, ${ }^{13}$ $\alpha=[\mathrm{R}(100 \mathrm{~V})-\mathrm{R}(0 \mathrm{~V})] /[\mathrm{R}(500 \mathrm{~V})-\mathrm{R}(400 \mathrm{~V})]$ should be unity. As calculated from the data in Fig. 3(a), $\alpha$ for PSMO with a thickness of $30 \mathrm{~nm}$ is $\sim 2.4$ at $72 \mathrm{~K}$. This means a significant violation from a linear $\Delta \mathrm{R} / \mathrm{R}$ vs $\mathrm{V}$ relation.

A qualitative comparison of nonlinearity could be made by normalizing the resistance modulation, $\delta_{\mathrm{n}}=\delta(\mathrm{V}) /$ $\delta(500 \mathrm{~V})$, as shown in Fig. 4(a). To get some insights, we use two quantities to characterize the nonlinearity. The first is the additional area under the measured $\delta_{\mathrm{n}} \mathrm{vs} \mathrm{V}$ curve [compared with that under a straight $\delta_{\mathrm{n}}-\mathrm{V}$ relation, see the example in Fig.4(a)], $\Delta s=0.2 \sum_{i=1}^{5} \delta_{n}(100 i)-0.6$. Here, for convenience, the voltage was normalized by $500 \mathrm{~V}$. The second is the standard deviation (deviation from a linear $\delta_{\mathrm{n}} \mathrm{vs} \mathrm{V}$ curve), $\sigma=\sqrt{\sum_{i=1}^{5}\left[\delta_{n}(100 i)-0.2 i\right]^{2}}$. It is obvious that for a linear $\mathrm{R}$ vs $\mathrm{V}$ relation, both $\Delta \mathrm{s}$ and $\sigma$ should be 0 . The determined $\Delta \mathrm{s}$ and $\sigma$ as a function of temperature for all PSMO films (when the voltage is decreased from $500 \mathrm{~V}$ to $0 \mathrm{~V}$ with a step of $100 \mathrm{~V}$ ) are shown in Figs. 4(c) and 4(d), respectively. For reference, the $|\Delta \mathrm{R}| / \mathrm{R}$ vs $\mathrm{T}$ curves are displayed in Fig. 4(b). Strikingly, for all films, the evolutions of
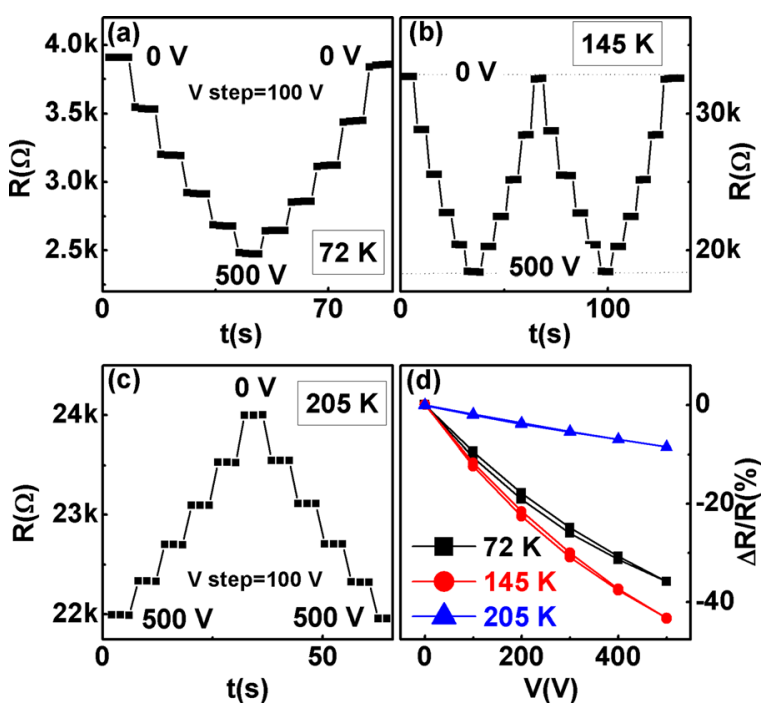

FIG. 3. (a)-(c) Resistance as a function of time while cycling voltages (between $500 \mathrm{~V}$ and $0 \mathrm{~V}$ ) for PSMO $(30 \mathrm{~nm})$ at $72 \mathrm{~K}(\mathrm{a}), 145 \mathrm{~K}(\mathrm{~b})$, and $205 \mathrm{~K}$ (c). The voltage step is $100 \mathrm{~V}$; (d) The dependence of relative resistance change on bias voltage deduced from (a)-(c). 

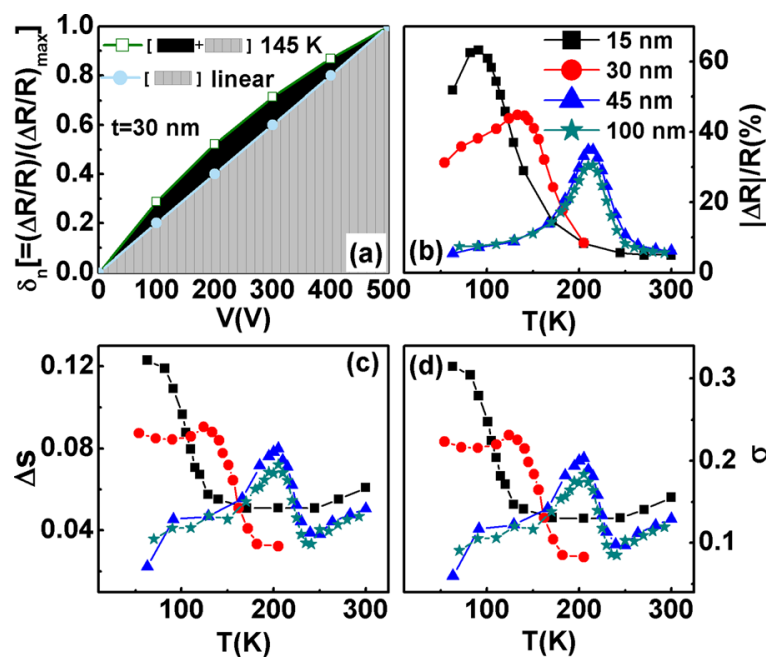

FIG. 4. (a) Normalized resistance modulation, $\delta_{\mathrm{n}}(\mathrm{V})=\delta(\mathrm{V}) / \delta(500 \mathrm{~V})$, as a function of applied voltages when the voltage was decreased from $500 \mathrm{~V}$ to $0 \mathrm{~V}$ with a step of $100 \mathrm{~V}(\mathrm{PSMO}, \mathrm{t}=30 \mathrm{~nm}, \mathrm{~T}=145 \mathrm{~K})$. For comparison, a linear dependence is shown; (b)-(d) Temperature dependence of resistance modulation $|\Delta \mathrm{R}| / \mathrm{R}(\mathrm{b})$, area difference $\Delta \mathrm{s}$ (c), and standard deviation $\sigma$ (d) for PSMO with different thicknesses.

$\Delta \mathrm{s}$ and $\sigma$ with temperature mimic that of corresponding resistance modulation $|\Delta R| / R$, signifying a close relation between them. As can be seen from Fig. 3(d), there is slight hysteresis in $\Delta \mathrm{R} / \mathrm{R}$ vs $\mathrm{V}$ curves. This means that the values of $\Delta \mathrm{s}$ and $\sigma$ obtained from $\Delta \mathrm{R} / \mathrm{R}$ vs $\mathrm{V}$ curves as the applied voltage increases from $0 \mathrm{~V}$ to $500 \mathrm{~V}$ should be different. We also calculated $\Delta \mathrm{s}$ and $\sigma$ from these $\Delta \mathrm{R} / \mathrm{R}$ vs $\mathrm{V}$ curves. Although there are slight differences in values, the temperature dependences are similar to those shown in Figs. 4(c) and 4(d). It is noted that when a new field was applied, there were small drifts (see Fig. 3), which are probably due to the relaxation behavior of PMN-PT. ${ }^{18,19}$ The extrinsic nonlinearity that caused by these drifts should have little influence on our results. Compared with the resistance modulation induced by the change of voltage, the overall drift for each applied field is quite small. More importantly, the nonlinearity depends on the relative rather than the absolute value of resistance modulation. The fixed duration of the voltage step within one voltage cycle in our experiments reduced the extrinsic nonlinearity that caused by these drifts. It is obviously premature to rule out the possibility of nonlinear converse piezoelectric effects as an origin of observed nonlinearity in $\Delta \mathrm{R} / \mathrm{R}$ vs $\mathrm{V}$ curves. ${ }^{18,23}$ However, by comparing the temperature dependences of nonlinearity for all samples, it is reasonable to conclude that PMN-PT should make very little contribution to the nonlinearity near $\mathrm{T}_{\mathrm{P} .}{ }^{18}$ The close correlation between the increased nonlinearity and the transition from metal to insulator suggests that the enhanced nonlinear- ity near $T_{P}$ should originate from the phase competition in PSMO films.

To conclude, the effects of reversible strain in PSMO thin films with various thicknesses were investigated by using a PSMO/PMN-PT structure. For the PSMO with a thickness of $15 \mathrm{~nm}$, when an electric field of $\mathrm{E}=10 \mathrm{kV} / \mathrm{cm}$ was applied to PMN-PT, an enhancement of $17 \mathrm{~K}$ in $\mathrm{T}_{\mathrm{P}}$ and a reduction of $\sim 70 \%$ in resistance were achieved. For all samples, the resistance did not always change linearly with applied voltage. We use two quantities, area difference and standard deviation, to characterize the nonlinearity. The nonlinearity, as well as the magnitude, of resistance modulation was strongly correlated to the metal-insulator transition. These results suggest that phase competition significantly alters the characteristics of strain responses.

This work has been supported by a grant of the Research Grant Council of Hong Kong (Project No. HKU 702409P).

${ }^{1}$ E. Dagotto, T. Hotta, and A. Moreo, Phys. Rep. 344, 1 (2001).
${ }^{2}$ M. B. Salamon and M. Jaime, Rev. Mod. Phys. 73, 583 (2001).

${ }^{2}$ M. B. Salamon and M. Jaime, Rev. Mod. Phys.
${ }^{3}$ Y. Tokura, Rep. Prog. Phys. 69, 797 (2006).

${ }^{4}$ R. von Helmolt, J. Wecker, B. Holzapfel, L. Schultz, and K. Samwer, Phys. Rev. Lett. 71, 2331 (1993).

${ }^{5}$ S. Jin, T. H. Tiefel, M. McCormack, R. A. Fastnacht, R. Ramesh, and L. H. Chen, Science 264, 413 (1994).

${ }^{6}$ W. Prellier, A. Biswas, M. Rajeswari, T. Venkatesan, and R. L. Greene, Appl. Phys. Lett. 75, 397 (1999).

${ }^{7}$ C. Adamo, X. Ke, H. Q. Wang, H. L. Xin, T. Heeg, M. E. Hawley, W. Zander, J. Schubert, P. Schiffer, D. A. Muller, L. Maritato, and D. G. Schlom, Appl. Phys. Lett. 95, 112504 (2009).

${ }^{8}$ F. Yang, N. Kemik, M. D. Biegalski, H. M. Christen, E. Arenholz, and Y. Takamura, Appl. Phys. Lett. 97, 092503 (2010).

${ }^{9}$ P. Murugavel, J. H. Lee, J. G. Yoon, T. W. Noh, J. S. Chung, M. Heu, and S. Yoon, Appl. Phys. Lett. 82, 1908 (2003).

${ }^{10}$ D. Dale, A. Fleet, J. D. Brock, and Y. Suzuki, Appl. Phys. Lett. 82, 3725 (2003).

${ }^{11}$ C. Thiele, K. Dörr, S. Fahler, L. Schultz, D. C. Meyer, A. A. Levin, and P. Paufler, Appl. Phys. Lett. 87, 262502 (2005).

${ }^{12}$ C. Thiele, K. Dörr, O. Bilani, J. Rödel, and L. Schultz, Phys. Rev. B 75, 054408 (2007).

${ }^{13}$ R. K. Zheng, Y. Wang, H. L. W. Chan, C. L. Choy, and H. S. Luo, Phys. Rev. B 75, 212102 (2007).

${ }^{14}$ R. K. Zheng, Y. Wang, H. L. W. Chan, C. L. Choy, and H. S. Luo, Appl. Phys. Lett. 92, 082908 (2008).

${ }^{15}$ M. C. Dekker, A. D. Rata, K. Boldyreva, S. Oswald, L. Schultz, and K. Dörr, Phys. Rev. B 80, 144402 (2009).

${ }^{16}$ E. J. Guo, J. Gao, and H. B. Lu, Appl. Phys. Lett. 98, 081903 (2011).

${ }^{17}$ R. K. Zheng, H. U. Habermeier, H. L. W. Chan, C. L. Choy, and H. S. Luo, Phys. Rev. B 80, 104433 (2009).

${ }^{18}$ A. Herklotz, J. D. Plumhof, A. Rastelli, O. G. Schmidt, L. Schultz, and K. Dörr, J. Appl. Phys. 108, 094101 (2010).

${ }^{19}$ A. A. Levin, A. I. Pommrich, T. Weissbach, D. C. Meyer, and O. BilaniZeneli, J. Appl. Phys. 103, 054102 (2008).

${ }^{20}$ L. P. Chen and J. Gao, Euro. Phys. Lett. 93, 47009 (2011).

${ }^{21}$ M. D. Biegalski, K. Dörr, D. H. Kim, and H. M. Christen, Appl. Phys. Lett. 96, 151905 (2010).

${ }^{22}$ J. Dvorak, Y. U. Idzerda, S. B. Ogale, S. Shinde, T. Wu, T. Venkatesan, R. Godfrey, and R. Ramesh, J. Appl. Phys. 97, $10 \mathrm{C} 102$ (2005).

${ }^{23}$ S.-E. Park and T. R. Shrout, J. Appl. Phys. 82, 1804 (1997). 